IOS Press

\title{
The "EyeCane", a new electronic travel aid for the blind: Technology, behavior \& swift learning
}

\author{
Shachar Maidenbaum ${ }^{\mathrm{a}, 1}$, Shlomi Hanassy ${ }^{\mathrm{a}, 1}$, Sami Abboud ${ }^{\mathrm{a}}$, Galit Buchs ${ }^{\mathrm{c}}$, Daniel-Robert Chebat ${ }^{\mathrm{a}, \mathrm{b}}$, \\ Shelly Levy-Tzedek ${ }^{\mathrm{a}, \mathrm{b}}$ and Amir Amedi $\mathrm{i}^{\mathrm{a}, \mathrm{b}, \mathrm{c}, *}$ \\ ${ }^{a}$ Department of Medical Neurobiology, Institute for Medical Research Israel-Canada, Faculty of Medicine, \\ The Hebrew University of Jerusalem, Jerusalem, Israel \\ ${ }^{\mathrm{b}}$ The Edmond and Lily Safra Center for Brain Research, The Hebrew University of Jerusalem, Jerusalem, Israel \\ ${ }^{\mathrm{c}}$ Department of Cognitive Science, The Hebrew University of Jerusalem, Jerusalem, Israel
}

\begin{abstract}
.
Purpose: Independent mobility is one of the most pressing problems facing people who are blind. We present the EyeCane, a new mobility aid aimed at increasing perception of environment beyond what is provided by the traditional White Cane for tasks such as distance estimation, navigation and obstacle detection.

Methods: The "EyeCane" enhances the traditional White Cane by using tactile and auditory output to increase detectable distance and angles. It circumvents the technical pitfalls of other devices, such as weight, short battery life, complex interface schemes, and slow learning curve. It implements multiple beams to enables detection of obstacles at different heights, and narrow beams to provide active sensing that can potentially increase the user's spatial perception of the environment. Participants were tasked with using the EyeCane for several basic tasks with minimal training.

Results: Blind and blindfolded-sighted participants were able to use the EyeCane successfully for distance estimation, simple navigation and simple obstacle detection after only several minutes of training.

Conclusions: These results demonstrate the EyeCane's potential for mobility rehabilitation. The short training time is especially important since available mobility training resources are limited, not always available, and can be quite expensive and/or entail long waiting periods.
\end{abstract}

Keywords: Blind, mobility, rehabilitation, sensory substitution, SSD, active sensing

\section{Introduction}

The challenges involved in independent mobility such as navigation, obstacle avoidance and distance estimation constitute some of the greatest difficulties facing the blind and visually impaired in their

\footnotetext{
${ }^{1}$ These authors contributed equally.

*Corresponding author: Amir Amedi, Ph.D., Dept. of Medical Neurobiology - The Institute for Medical Research Israel-Canada, Faculty of Medicine, The Hebrew University of Jerusalem, Jerusalem 91220, Israel. Tel.: +972 2675 7259; Fax: +972 2675 8602; E-mail: amir.amedi@ekmd.huji.ac.il; http://brain.huji.ac.il/.
}

everyday lives (Jacobson et al., 1993; Douglas et al., 2006; Quinones et al., 2011), and often cause them to lose their bearings (White et al., 2008) or suffer injury (Manduchi and Kurniawan, 2010; 2011) even when using traditional aids such as the White Cane. This occurs despite the excellent spatial memory of the blind, which is in some cases superior to that of the sighted (Loomis and Klatzky, 2001; Raz et al., 2007), because it is far more difficult to avoid obstacles and create a mental map of one's environment in the absence of vision (Loomis and Klatzky, 2001; McVea and Pearson, 2009). 
Traditionally, these challenges have been tackled using a White Cane. However, only part of the blind population is currently using White Canes (although the best of our knowledge there are no world-wide statistics, see Russell et al., 1997; Marson 2003; Gold and Simson, 2005; Christy and Nirmalan, 2006 for some individual country surveys which report White Cane use ranging from $17 \%$ to $50 \%$ ). Furthermore, only $15 \%$ of the visually impaired and/or blind including White Cane users, leave their homes at least once a day (Douglas et al., 2006; Manduchi and Kurniawan, 2011). There are multiple reasons why many blind people avoid the use of the White Cane, but the primary factors are (1) fear of striking people or fragile objects with it, (2) collisions with obstacles at a height that the White Cane does not detect and (3) the social stigma among some of the blind and primarily the late-blind (though it should be noted that many others embrace this differentiation positively, and use the White Cane to draw attention to themselves as blind deliberately; see Pavey, Dodgson et al., 2009 and Discussion). In some cases even only one of these reasons may prompt an individual to avoid or rarely use a White Cane.

One promising approach to ameliorating the White Cane is to augment or replace the physical cane with a sensor and an alternative output interface system. Such a device is known as a "Virtual Cane", or an "Electronic Travel Aid" (ETA). Virtual canes solve several of the main problems of White Canes - they are less obtrusive because there are no collisions between the ETA and people or fragile objects in the vicinity, their reach is longer (several meters vs. $\sim 1 \mathrm{~m}$ ), and their weight and shape enable them to be easily pointed at different heights, thus providing protection from higher obstacles.

Many ETAs have been developed over the years. Early examples include the Sonic Torch (Kay, 1964) and the PathSounder (Russell, 1965). Other notable examples were the UltraCane (Hoyle, 2008) and the Télétact (Farcy, 2002; for recent reviews see Roentgen et al., 2008; Dakopoulos and Bourbakis, 2010; Liu et al., 2010). The initial reception of these devices by blind users in experimental conditions was positive (Roentgen et al., 2008). Unfortunately however, none have matured into widely used tools or have gained acceptance in the blind community because of the many problems involved in using them in real-world use-cases such as their size, weight, battery life, reliability, ease of use, cost, interference with other senses and primarily the time required to master them even on a basic level, which is the parameter we focus on here (Dakopoulos, 2009; Dakopoulos and Bourbakis, 2010; Quinones et al., 2011).

A different approach involves the use of more generic devices known as sensory substitution devices (SSDs) which are intended to convey visual information to the blind, and can then be used for mobility purposes as well. See (Bach-y-Rita, 1972; Meijer, 1992; Capelle et al., 1998; Hanneton et al., 2010; Williams et al., 2011; Kärcher et al., 2012; LevyTzedek et al., 2012; Abboud et al., 2014) for examples of such devices and (Maidenbaum and Amedi, 2012; Maidenbaum, 2014) for recent reviews on the use of such devices for visual rehabilitation. However, here as well these devices have so far failed to be adopted widely by the blind community (despite some remarkable success reported by individuals for complex daily life tasks; see Ward and Meijer, 2010; links in supplementary data). Some of the main barriers to adoption appear to be the complexity of the input signal and the long training time required to master them.

Any device that attempts to either augment or replace the White Cane should enable better independent mobility in natural environments than provided by the White Cane alone, including reduction of the number of collisions (Manduchi and Kurniawan, 2010), while at the same time being both simple and intuitive to use (Roentgen et al., 2008; Dakopoulos and Bourbakis, 2010).

To tackle these issues, we developed the "EyeCane" (Amedi and Hanassy, 2012), a novel ETA for the blind that uses multiple infra-red sensors aimed at different directions with tactile and audio output. The EyeCane has a range of $5 \mathrm{~m}$, is small $(4 \times 6 \times 12 \mathrm{~cm}$, similar in size to a small cellphone), lightweight $(\sim 100 \mathrm{~g})$, has long battery life (can go for a day without charging, and is easily rechargeable), was designed so that it can be manufactured at low cost, and has an output signal which is intuitive to the user.

In this paper we outline the features of the EyeCane device, and explore its capabilities in a series of three experiments designed to test basic everyday tasks, and explore both the feasibility and intuitiveness of its use with emphasis on its use after relatively short, minimal training.

Experiment 1 (Distance Estimation) explored the participants' ability to estimate distances using the device. Experiment 2 (Navigation) explored the participants' ability to interact with a simple natural environment unfamiliar to them. Experiment 3 (Obsta- 
a
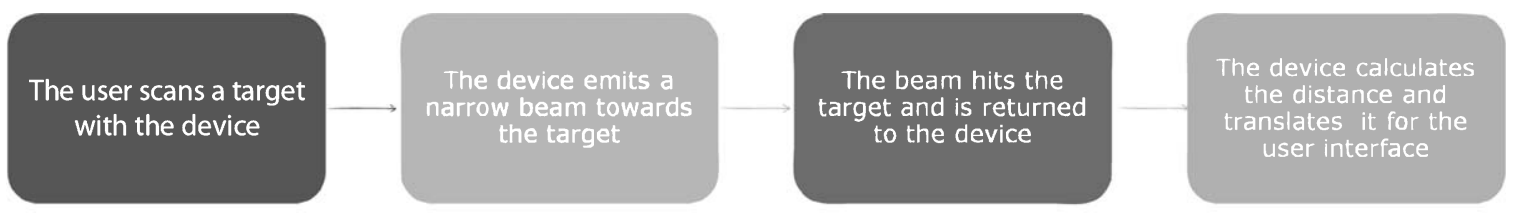

b
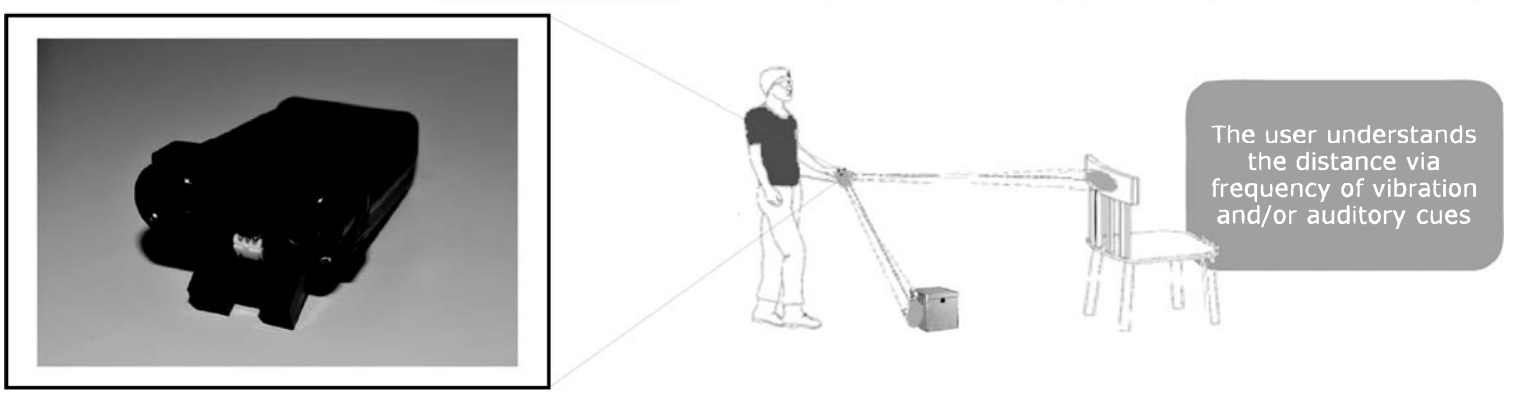

Fig. 1. The EyeCane: (a) A flow chart depicting the use of the device and an illustration of a user. Note the two sensor beams, one pointing directly ahead, and one pointing towards the ground for obstacle detection. (b) Photo of the "EyeCane".

cle Detection) used the same setup to explore the effect of added natural obstacles.

\section{Materials and methods}

\subsection{Ethics}

All of the experiments were approved by the Hebrew University's ethics committee, and all participants signed informed consent forms. Blind and blindfoldedsighted participants were accompanied at all times by an experimenter dedicated to guaranteeing their safety.

\subsection{Statistical analysis}

Significance was determined at the level of $p<0.05$ after correction for multiple comparisons. SD is given together with mean values in the following format "mean \pm SD (range:lowest-highest)".

\subsection{Overall participants}

A total of 43 participants (38 sighted blindfolded, 18 male, 38 right handed) took part in these experiments, 10 in experiment 1,13 in experiment 2 and 12 of these 13 in experiment 3 . Eleven additional blindfolded-sighted participants (matched to the 11 sighted participants in experiments 2-3) participated in control-experiment 1 for experiment 2, and 9 additional blindfolded-sighted participants participated in control-experiment 2 for experiment 2 .

\subsection{The "EyeCane"-Device description}

We developed the "EyeCane", an ETA for the blind which translates point-distance information into auditory and tactile cues (instructions and a picture of the device can be seen in Fig. 1, a video showing practical use can be found in appendix 1).

The device provides the user with distance information simultaneously from two different directions: directly ahead for long distance $(5 \mathrm{~m})$ perception of the environment and detection of waist height obstacles, and towards the ground at a $45^{\circ}$ angle for detecting nearby ground level obstacles $(1.5 \mathrm{~m})$. This information is conveyed to the user using different auditory frequencies and by different tactile actuators (in contact with the thumb for ground level obstacles and with the wrist for waist level obstacles).

Each of these directions is detected via a dedicated pair of infra-red (IR) emitters and sensors (Sharp GP2D12, GP2Y0A710). The emitters, which are photodiodes with concentrating lenses, emit narrow directional beams $\left(<5^{\circ}\right)$ in the direction at which they are aimed, at different frequency ranges, thus enabling their use for two separate distances and directions in parallel. The reflected signal is then detected by phototransistors sensitive to the emitted IR wavelengths, which were previously tested on different surfaces and lighting conditions (Innet and Ritnoom, 2008). The phototransistor signals in turn modify the baseline voltage in the electrical circuit, translating the distance from the detected object into a DC voltage signal. This DCvoltage signal is translated in real-time $(>50 \mathrm{~Hz})$ into 
sound frequencies and/or vibration amplitudes and frequencies, enabling instantaneous feedback to the user such that the closer an object is to the user the higher the frequency of the auditory cues and the stronger the vibration. The sensitivity range of the distances can be calibrated via modulation-resistors, enabling flexibility for user preference and different scenarios.

This design differs conceptually from most previous devices in two main ways. First, we chose to use focused beams, thus adopting an active sensing "spotlight" approach for obstacle detection. This approach forces the user to constantly scan the environment but in return allows for the location of obstacles with greater accuracy and the acquisition of more information about them than is possible with wide-beam sensors. Another reason for this choice were the many growing body of results in recent years which has emphasized the importance of active sensing for perceiving the environment and the objects within it (see for example Lenay, Gapenne et al., 2003; Auvray et al., 2009; Horev et al., 2011).

The second is the use of multiple sensors aimed in different directions and with different distance sensitivities to simultaneously enable both detection of near-ground-level obstacles and waist-level obstacles several meters ahead, thus providing upper body protection (which is one of the main limitations of the White Cane), and a general perception of the upcoming five meters, such as locating open doors on the other side of a room.

These innovations join a series of design considerations, including decreased size $(4 \times 6 \times 12 \mathrm{~cm})$ and weight $(<100 \mathrm{~g})$ which enable it to be easily held and pointed at different targets while increasing battery life ( $>24 \mathrm{~h}$ of use). See Amedi and Hanassy, 2012 for more specific details and Roentgen, Gelderblom et al., 2008; Dakopoulos and Bourbakis, 2010; Liu, Liu et al., 2010 for these parameters in previous devices.

\section{Experiment 1 - Distance estimation experiment}

This experiment tested participants' ability to accurately estimate distances after less than 5 minutes of training. Participants were asked to report vocally the distance of a sheet of cardboard that was placed in front of them at 1, 1.5, 2, 2.5 and 3 meters. Participants were aware that the sheet would be placed at one of these five distances on every trial. Answers were noted by a member of the experimental team. The aim here was on exploring general distance estimation and quick intuitive use rather than on precision testing. Fifty trials were conducted in a semi-randomized order. To determine chance level, participants were requested to estimate the distance without the device on 10 additional trials to make sure that they were not relying on any other cues besides the device (such as echolocation or the breathing of the experimenters). This experimental chance value was set as the Control bar.

\subsection{Experimental setup}

Participants were comfortably seated at a long table while holding the device (see Fig. 2a). One experimenter moved the target sheet of cardboard $(69 \times 91 \mathrm{~cm}$, acting as a portable "wall") on each trial, and a second experimenter, seated beside the participant cued them when a new trial began (to avoid auditory localization of the target) and recorded their answer.

\subsection{Training}

During training, the sheet of cardboard was moved back and forth in front of each participant, with the different distances pointed out, to give participants a feel of the different outputs (for example, "The board is now 2 meters away, this is what an object at this distance feels like"). It should be noted that although learning to differentiate between near/far with the EyeCane is nearly instantaneous, mental calibration of the exact mapping of auditory-frequencies/vibrations to the exact distances seemed to take longer. The duration of training was less than 5 minutes for all participants.

\subsection{Participants}

Ten participants took part in this experiment; 7 sighted blindfolded participants (4 male, all righthanded) and 3 blind (1 male, all right handed).

\subsection{Results}

The Control bar established by the control experiment, in which distances were estimated without the device, was $22 \%$, which was indeed close to the expected random chance level. This showed that participants were indeed guessing at random and not relying on external cues. 

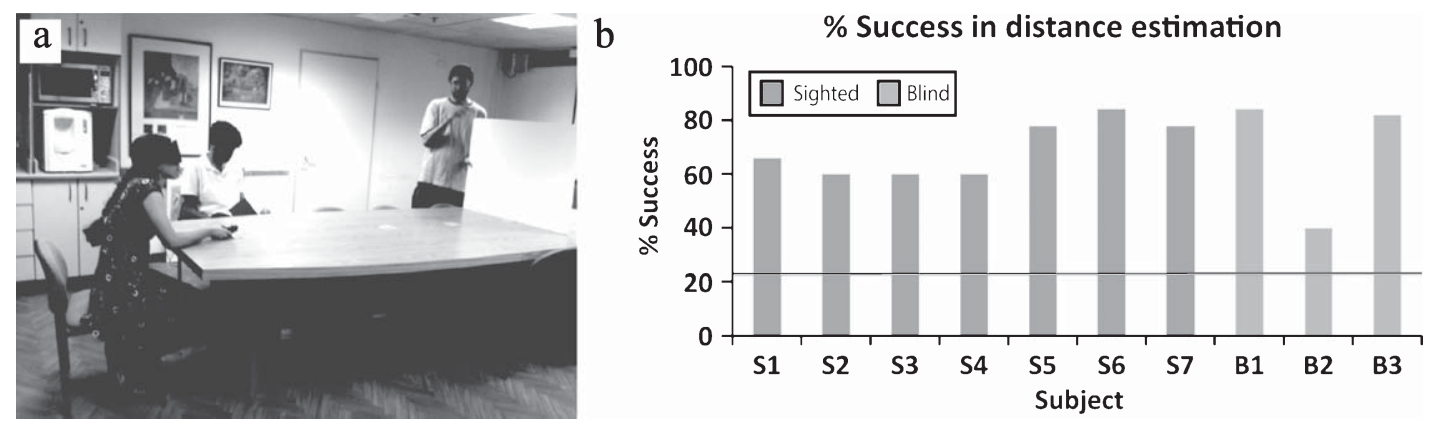

Fig. 2. Distance estimation: (a) The experimental setup for experiment 1 , distance estimation. (b) Results from experiment 1 , distance estimation, showing that all sighted (left) and all blind participants (right) performed well above control level after less than 5 minutes of training. Control level (line) was determined by a block of 10 control trials at a random point during the experiment in which each participant had the device turned off. Note that each column denotes a single participant.

All participants performed significantly above Control $(p<7 \mathrm{e}-7$, standard $t$-test). The group success rate was $69.4 \% \pm 9.5 \%$ for the sighted participants, and $68.6 \pm 24.8 \%$ for the blind participants.

It should be noted that of the blind participants, B1 and B3 answered correctly on $84.3 \%$ and $82 \%$ of the trials respectively. The third blind participant, B2, reported great difficulty understanding the concept of distances in meters, and succeeded in only $40 \%$ of trials, far lower than the other participants but still higher than Control (Fig. 2b).

\section{Experiment 2 - Navigation}

This experiment tested participants' ability to use the EyeCane in an unfamiliar natural environment. Participants were asked to complete a walk down a winding corridor (Fig. 3a) while avoiding collisions with the walls. Each participant carried outfour trials. After trials $1 \& 4$, participants, while still blindfolded, were asked to draw the corridor they had walked through (see Fig. 3b-c). As controls, a separate group of participants walked the corridor without the device (control experiment 1) and a second group walked this same corridor using a White Cane fitted to their height (control experiment 2).

\subsection{Experimental setup}

The navigation and control experiments were conducted in a real world natural environment, a corridor in the Hebrew University of Jerusalem unfamiliar to the participants. Sighted participants remained blind- folded at all times in the experimental area and throughout the duration of the training and experiments. In experiments 2-3 we use the term "collisions" to refer to any contact of the participant's body or the device with the walls or obstacles.

\subsection{Training}

At the beginning of this experiment participants received basic training on the device. This included a basic explanation of how the device works and how to use it, walking towards and away from a wall to obtain a feeling for how the various distances are translated into different sound frequencies and vibrations, and pointing the device at different objects in their vicinity. The duration of training was less than 3 minutes. For a video demonstration of a similar training session see Appendix 1.

\subsection{Participants}

Thirteen participants (11 sighted, 5 male, aged 24 (21-54), 9 right-handed) took part in this experiment. An additional group of 11 blindfolded sighted participants took part in control experiment 1 (matched by gender and age to the 11 sighted participants), and 9 additional blindfolded-sighted participants participated in control experiment 2 (fitted with a White-Cane for their height).

\subsection{Results}

Scores for the blind participants were similar to those of the sighted, and were therefore grouped with 


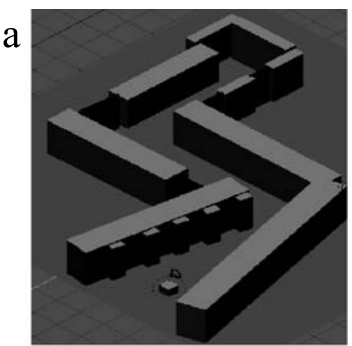

b

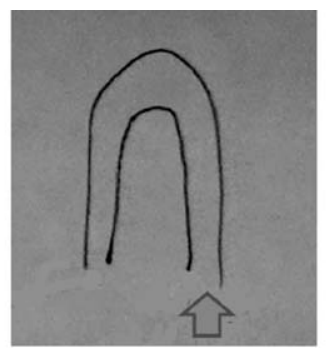

C

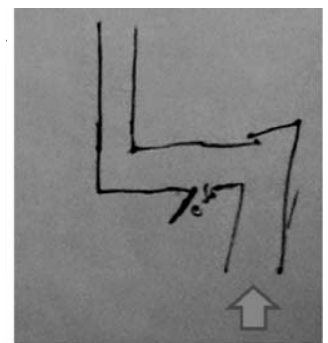

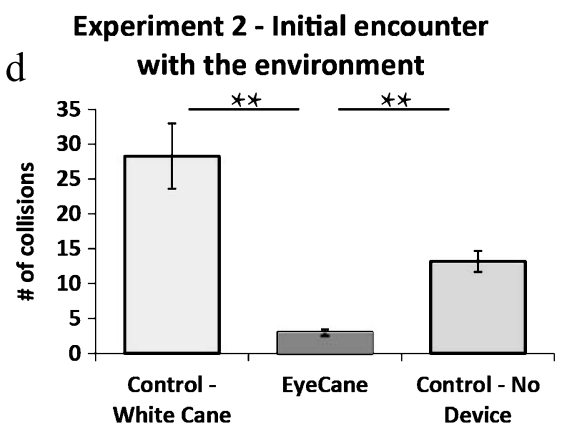
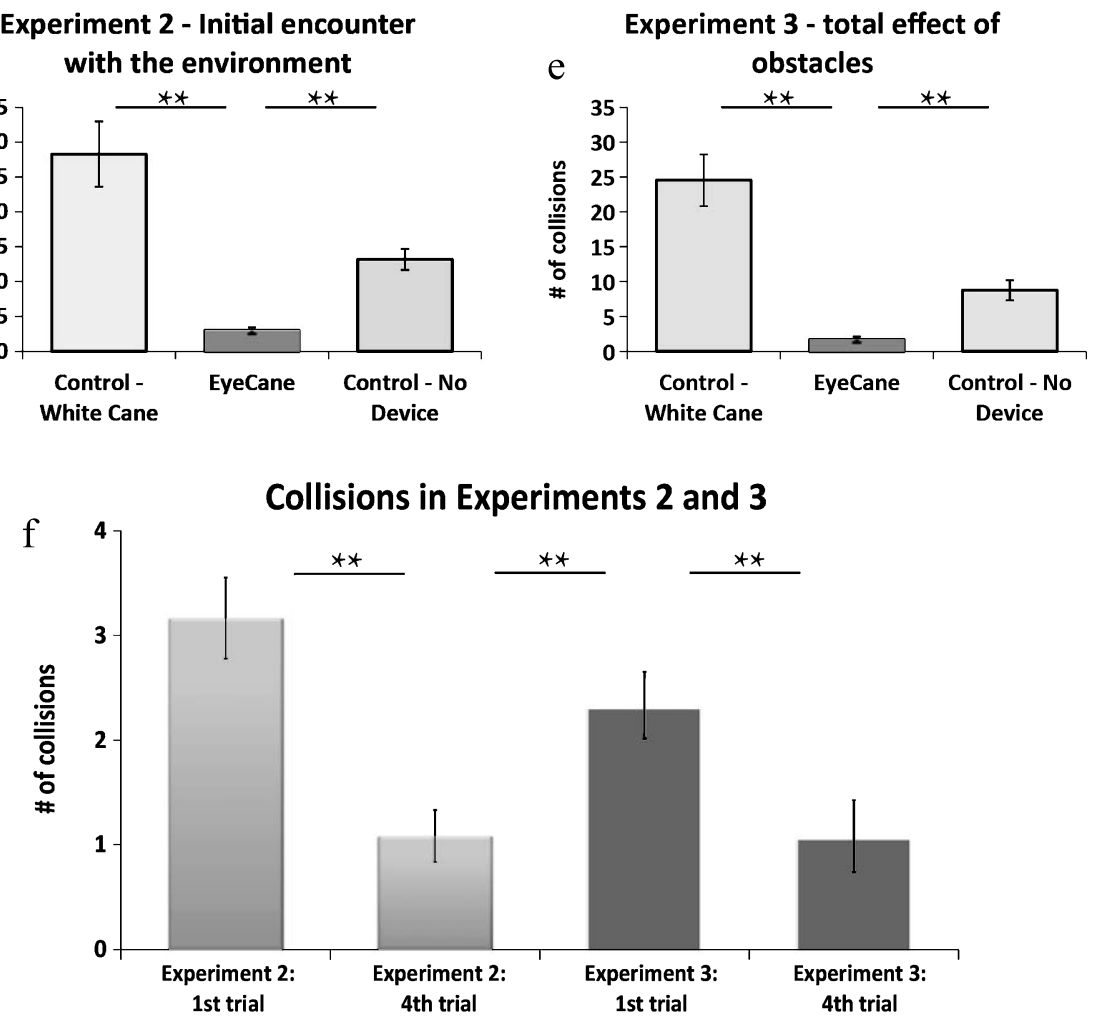

Fig. 3. Navigation and Obstacle detection: (a) A graphical reconstruction of the experimental environment. (b-c) Participant drawings of the route after trial 1 (in b) and trial 4 (in c) of experiment 2. Blue arrows mark the starting point of the participants' drawing. (d) Experiment 2 results comparing the participants' first trial showing that there were significantly fewer collisions with the walls of the corridor when using the EyeCane than when using a White Cane or when not using any assistive device. (e) Experiment 3 results, comparing all the participants' trials showing that there were significantly fewer collisions with the walls of the corridor and with the obstacles when using the EyeCane than when using a White Cane or when not using any assistive device. (f) Experiment 2 and 3 results, showing a highly significant improvement during experiment 2 (4th trial significantly better than 1st), a drop in scores after the introduction of obstacles in Experiment 3 (4th trial of experiment 2 significantly better than 1 st of experiment 3 ) and a return to the same level by the final trial of experiment 3 (4th trial of experiment 3 significantly better than 1 st of experiment 3 , and similar to 4 th of experiment 2 ).

them for these comparisons ( $p=0.4$ for average $t$-test comparison between the groups for each of the runs). Nearly all participants succeeded in navigating to the end of the corridor on all trials $(96.2 \% \pm 2.6 \%(3-4))$, with a gradual reduction in the number of collisions. Two of the blindfolded-sighted failed on a single trial by mistakenly returning to the starting point. Control group 2 using the White Cane had a somewhat lower success rate although it did not differ significantly from the experimental group $(92 \pm 17 \%(2-4), p=0.17)$.

On the first trial, participants had $3 \pm 1.4(0-5)$ collisions, which was significantly better than control group 1 ( $p<8 \mathrm{e}-6$, standard $t$-test) who attempted to walk down the same corridor for the first time blindfolded 
without any device and had $13.1 \pm 5.2(7-25)$ collisions (Fig. 3d). This was also significantly better than control group 2 with $28.2 \pm 11.5$ (20-46) collisions $(p<1.6 \mathrm{e}-3$, standard $t$-test), who using a White Cane had nearly ten times as many contacts with the walls. This difference remained significant on the 4 th trial as well with $23.1 \pm 6.8(18-32)$ collisions $(p<1.3 \mathrm{e}-4$, standard $t$-test).

Participants improved significantly between the first and fourth trial, from a mean of $3.1 \pm 1.3(0-5)$ collisions per trial to a mean of $1 \pm 0.8(0-2)$ collisions per trial $(p<4 \mathrm{e}-5$, paired $t$-test; Fig. 3f). By the end of the 4th trial all participants were easily able to draw and describe the corridor they had walked down (example in Fig. 3b-c. No interesting result was found in these drawings beyond the reported ability and subjective assessment by the experimenters).

\section{Experiment 3 - Obstacle avoidance}

In a continuation of experiment 2 , the same participants were tested with the device in the same natural environment with added obstacles at varying locations. As in experiment 2, on each of four trials, participants were asked to make their way along the winding corridor while focusing on completing the route while avoiding collisions with obstacles or walls. Additionally, participants were requested to stop and point out obstacles (vocally and by physically pointing at them) if they identified them. There were three obstacles on each trial, but this number was not divulged to the participants. No training was added to that of experiment 2. As controls, each participant had one trial without the device at the end of the experiment and the participants in control experiment 2 carried out all of the trials in this experiment as well using a White Cane.

\subsection{Experimental setup}

As in experiment 2, with the addition of 3 natural obstacles (chair, coffee dispenser and person, randomly relocated for each trial).

\subsection{Training}

Followed experiment 2 directly, with no additional training.

\subsection{Participants}

As in experiment 2, except for sighted participant OL who did not take part in this experiment. Control group 2, using the White Cane, performed this experiment as well.

\subsection{Results}

All participants were able to successfully navigate to the end of the corridor without returning to the starting point on all trials, gradually reducing the number of collisions. In comparison, the participants in control group 2 using the White Cane had a success rate of only $85.7 \pm 35 \%(p<0.04)$.

The number of collisions per trial dropped significantly between the first and fourth trials of experiment 3 ( $p<8 \mathrm{E}-3$, Paired $t$-test), from a mean of $2.3 \pm 1.1$ $(0-4)$ collisions per trial to a mean of $1 \pm 1.2(0-4)$ collisions per trial (Fig. 3f).

The introduction of obstacles in experiment 3 caused a significant increase in the number of collisions $(p<4 \mathrm{E}-4$, Paired $t$-test, when comparing trial 4 of experiment 2 and trial 1 of experiment 3 ), but in the following trials the participants returned to their previous levels ( $p=0.5$, Paired $t$-test, when comparing trial 4 of experiment 2 and trial 4 of experiment 3 ), despite the increased potential for collisions in Experiment 3.

Participants' performance during the control trial in which they were not using the EyeCane $(10.2 \pm 4.1$ (5-20) collisions) was significantly worse both compared to the first $(p<2.7 \mathrm{E}-4$, paired $t$-test $)$ and fourth $(p<9.7 \mathrm{E}-5$, paired $t$-test) trials, indicating that the EyeCane significantly helped the participants avoid collisions even on their first attempt (Fig. 3e).

Participants in control experiment 2, who used the White Cane, had a significantly lower success rate (only $85.7 \pm 35 \%, p<0.04$ ), and had significantly more collisions both on their first trial $(27.8 \pm 9.8$ $(19-49 p<4 \mathrm{e}-4$, standard $t$-test) and on their last trial (19.2 \pm 9 (9-35 $p<1.6 \mathrm{e}-3$, standard $t$-test).

\section{Discussion}

We presented the "EyeCane" ETA, and showed that it can be used successfully for various tasks in real environments such as distance estimation, navigation in natural environments and obstacle detection even after less than 5 minutes of training. 
The EyeCane has the potential to enable blind users to both perceive their environment and avoid obstacles with an extremely fast learning curve. The blind users not only completed the above tasks, but were extremely enthusiastic about the device ("When can I take one home?" blind participant B1, "Maybe I can borrow it just for a few hours?" blind participant B2).

To show that the participants' results indeed stemmed from their use of the EyeCane, three of the control experiments compared participant performance without the use of any device to control for other forms of environmental perception such as subconscious echolocation or memory. Indeed, all three of these control experiments showed that without the device participants performed far worse.

The fourth control experiment compared the results in experiments 2-3 with the EyeCane vs. the traditional White Cane and found that success with the White Cane was lower, and that the number of contacts with the White Cane was significantly higher, often by an order of magnitude. This result is unsurprising since such contacts are inherent to proper use of the White Cane, whose function entails finding walls and obstacles by contact with them. This obtrusiveness is one of the main motivations behind the use of ETAs such as the EyeCane, as many users are uncomfortable with physically contacting their surroundings or potentially hitting other people or fragile objects.

\subsection{Comparison to other devices}

The EyeCane was designed to augment, or possibly even in the more distant future to replace, the traditional White Cane by adding information at greater distances (5 m) and more angles, and most importantly by eliminating the need for contacts between the cane and the user's surroundings inherent to its use in cluttered or indoor environments.

Compared to other ETAs that also attempt to ameliorate the White Cane, the EyeCane has the following distinct combination of attributes. (1) It uses narrow beams, enabling exact information about the user's surroundings and provides the advantages of active sensing, though at the cost of requiring the user to constantly scan the environment. (2) It uses multiple beams at different distances and directions to enable both ground and waist-high obstacle detection. (3) It avoids many pitfalls which have plagued previous devices such as response-lags, battery-life, cost, weight, simplicity, unobtrusiveness and size (for reviews see Roentgen et al., 2008; Dakopoulos and Bourbakis, 2010; Liu et al., 2010).

A direct behavioral comparison to other ETAs is difficult, as most publications over the past decade mainly provide technical information with few detailed behavioral results. Even works which do report such experiments tend to report limited behavioral tasks in controlled environments and often did not include fully blind users (for example, see Roentgen et al., 2008; Jie et al., 2010; Froese et al., 2011). Thus, we do not claim to have proven behavioral advantages over these other devices, but merely to have demonstrated the successful use of the EyeCane and highlighted the differences and potential advantages of our approach. We give special emphasis on the level of performance after only several minutes of training as compared to hours or days in previous works.

Future standardization of the tests in this field to enable such behavioral comparison would constitute an important contribution, and several efforts have recently been made in this direction, such as the work of Nau et al. (2014) whose suggested tests we plan to perform as one of the next steps of evaluating the EyeCane.

Sensory Substitution Devices such as the vOICe (Meijer, 1992), EyeMusic (Levy-Tzedek et al., 2012; Levy-Tzedek et al., 2012) and TDU (Bach-y-Rita, 1972) do not directly convey distance information, but such information can be extracted indirectly from the general visual information they provide in a similar fashion to that of monocular depth vision. However, extracting this information is highly difficult, imprecise and can only be extracted by highly experienced users (Ward and Meijer, 2010; Chebat et al., 2011).

Since the "EyeCane" conveys only point-distance information, it is not planned for use as a stand-alone aid for the blind, but rather as part of an array of dedicated devices which should be used together. For example, it can enhance the White Cane with further distance and angles, or synergistically combine with SSDs such as the vOICe or TDU for shape and location information or the "EyeMusic" for shape, location and color information as suggested in Reich, 2012.

\subsection{Intuitiveness and training speed}

A key problem with many devices for the blind is the long training curve required for mastering them. One of the key results we show here is that even after less 
than five minutes of training participants were able to complete the tasks successfully. This result is a significant factor as regards the potential for user adoption of the EyeCane (Maidenbaum and Amedi, 2014).

\subsection{Sighted and blind participants}

In general, our results show that the blind and sighted-blindfolded participants behaved very similarly in our experiments. It would be interesting to assess whether these results hold true for a larger group of blind participants, especially in the distance estimation task where subject B2 performed very differently. It would also be worthwhile to comparatively explore the behavior of late blind and visually impaired participants.

\subsection{Natural environment}

Previous works have not always been successful in bridging the gap between success rates in ideal environments and actual performance in real-world conditions (Loomis, 2010). Thus, we chose to run experiments 2-3 in a natural environment with natural obstacles, rather than in dedicated ideal environments, thereby illustrating the EyeCane's ecological validity.

\subsection{Active sensing}

The EyeCane requires the user to actively explore the environment. This can be seen as a disadvantage, as it both requires the user to constantly scan with the device and may mean that an important object can be missed. However, the narrow beams give the user very high spatial resolution. Furthermore, such active sensing has been shown to play a major part in the perception of the environment (Lenay et al., 2003; Auvray et al., 2009; Froese et al., 2011), and better mimics the natural sensory process associated with spatial perception (visual saccades and tactile exploration in humans, and additional senses such as whisking in animals (Horev et al., 2011)). Although we did not compare directly to a passive sensing approach, we believe our results can be seen as another example of the advantages of this approach.

\subsection{Stigma}

One of the main reported problems with the White Cane is the stigma involved in its use. The EyeCane does not attempt to provide a full solution to this problem since although it is less obtrusive than the White Cane, it is still clearly visible. On the other hand, like other similar devices it does not have the cultural and emotional stigma associated with users, especially those in the process of losing their vision. It should be noted that for many other users a solution to the problem of stigma might not even be welcome, as others' awareness of the user's disability can also encourage them to help the user in ways which many in the blind population rely on implicitly or explicitly. A partial and limited solution to this problem may be not so much technological but rather social, since ETAs in general resemble wearable-computing devices, which nowadays are slowly becoming more accepted in society, as compared to the White Cane which clearly brands the user as disabled.

\subsection{Distance, distal attribution and environment perception}

By the end of experiments 2 and 3 the participants were able to describe and recreate the outline of the walls of the entire winding corridor, without ever being in physical contact with it beyond sporadic collisions. This was especially true for the blind participants, and thus shows that they were able to create a mental map of the environment.

The term Distal attribution describes how perception of vibrations on the surface of the palm of the hand changes with the knowledge that this perception refers to an external object in space (for review see Auvray et al., 2005), and is commonly observed in many SSDs (Renier and De Volder, 2010; Chebat et al., 2011). Similary, the EyeCane may also change this perception. This is especially true for blind participants, whose perception of distance and space beyond their peri-personal space is limited. One of the blind participants described the use of the "EyeCane" as follows: "I could feel the world stretching out before me. It was as if my hand could reach much further. As if the silent objects on the other side of the room were suddenly there" (Participant B3, during the distance estimation experiment).

In the future, the EyeCane and the swift learning of the transformation it provides for distance information could make it possible to explore different parameters of this process such as the relative effect of distance cues from different sensory modalities. 


\subsection{Virtual version, rehabilitation and neuroscience}

The EyeCane is not only applicable in the real world. We created a virtual version to examine its advantages in virtual environments for tasks such as navigation and shape recognition (Maidenbaum et al., 2012, 2013). Potentially, this will allow users to train safely at home on new environments before encountering them in the real world, thereby increasing the rehabilitation potential of the EyeCane.

In addition this virtual representation can be implemented during brain imaging experiments to research basic questions in the field of neuroscience such as the neural correlates of absolute distance and active navigation without vision.

\section{Conclusion}

We presented the EyeCane and showed its practical use for distance estimation, navigation and obstacle detection, which are all major tasks in mobility rehabilitation for the blind, and its advantages over the use of a White Cane alone for these tasks. Importantly, success was achieved after a minimal training time and in a natural environment, indicating the EyeCane's potential applications for practical rehabilitation.

\section{Acknowledgments}

The authors wish to thank Anatoly Shapochnikov for his help in designing and developing the device, and Avigail Beryozkin for her help in running some of the experiments. This work was supported by a European Research Council grant to AA (grant number 310809), the Charitable Gatsby Foundation, the James S. McDonnell Foundation scholar award (to AA; grant number 220020284), the Israel Science Foundation (grant number ISF 1684/08), the Edmond and Lily Safra Center for Brain Sciences (ELSC) Vision center (to AA, SL, DRC). SA was supported by a scholarship from the Israeli Ministry of Science.

\section{Disclaimer}

SH \& AA are co-authors of the patent (Amedi and Hanassy, 2012) which the EyeCane is an implementation of.

\section{References}

Amedi, A., \& Hanassy, S., (2012). Infra Red based devices for guiding blind and visually impaired persons, US Patent $2,012,090,114$.

Auvray, M., Hanneton, S., Lenay, C., \& O'Reagn, K. (2005). There is something out there: Distal attribution in sensory substitution, twenty years later. J Integr Neurosci, 4(04), 505-521.

Auvray, M., Lenay, C., \& Stewart, J. (2009). Perceptual interactions in a minimalist virtual environment. New Ideas Psychol, 27(1), $32-47$.

Abboud, S., Hanassy, S., Levy-Tzedek, S., Maidenbaum, S., \& Amedi, A. (2014) EyeMusic: Introducing a "visual" colorful experience for the blind using auditory sensory substitution. Restor Neurol Neuros, 32.2, 247-257.

Bach-y-Rita, P. (1972). Brain mechanisms in sensory substitution, New York: Academic Press.

Capelle, C., Trullemans, C., Arno, P., \& Veraart, C. (1998). A real-time experimental prototype for enhancement of vision rehabilitation using auditory substitution. IEEE Trans Biomed Eng, 45(10), 1279-1293.

Chebat, D.R., Schneider, F.C., Kupers, R., \& Ptito, M. (2011). Navigation with a sensory substitution device in congenitally blind individuals. NeuroReport, 22(7), 342.

Christy, B., \& Nirmalan, P.K. (2006). Acceptance of the long Cane by persons who are blind in south india. JVIB, 100(2), 115.

Dakopoulos, D. (2009). TYFLOS: A Wearable navigation prototype for blind \& visually impaired; design, modelling and experimental results. PhD Diss Wright State University.

Dakopoulos, D., \& Bourbakis, N.G. (2010). Wearable obstacle avoidance electronic travel aids for blind: A survey. IEEE Trans Syst Man Cybern, 40(1), 25-35.

Douglas, G., Corcoran, C., \& Pavey, S. (2006). Opinions and circumstances of visually impaired people in Great Britain: Report based on over 1000 interviews. Network 1000 Report, Visual Impairment Centre for Teaching and Research, University of Birmingham.

Farcy, R., \& Bellik, Y. (2002). Locomotion assistance for the blind. UAAT, Springer, 277-284.

Froese, T., McGann, M., Bigge, W., Spiers, A., \& Seth, A.K. (2011). The enactive torch: A new tool for the science of perception. IEEE Trans Hapt, 5(4), 365-375.

Gold, D., \& Simson, H. (2005). Identifying the needs of people in Canada who are blind or visually impaired: Preliminary results of a nation-wide study. International Congress Series, 1282, 139-142. Elsevier.

Hanneton, S., Auvray, M., \& Durette, B. (2010). The Vibe: A versatile vision-to-audition sensory substitution device. Appl Bionics Biomech, 7(4), 269-276.

Horev, G., Saig, A., Knutsen, P.M., Pietr, M., Yu, C., \& Ahissar, E. (2011). Motor-sensory convergence in object localization: A comparative study in rats and humans. Phil Trans $R$ Soc B: Biological Sciences, 366(1581), 3070-3076.

Hoyle, B., \& Waters, D. (2008). Mobility AT: The Batcane (UltraCane). In Assistive technology for visually impaired and blind people (pp. 209-229). Springer, London. 
Innet, S., \& Ritnoom, N. (2008). An application of infrared sensors for electronic white stick. In Intelligent Signal Processing and Communications Systems, 2008. ISPACS 2008 proceedings (pp. $1-4)$.

Jacobson, W.H. (1993). The art and science of teaching orientation and mobility to persons with visual impairments. $A F B$.

Jie, X., Xiaochi, W., \& Zhigang, F. (2010). Research and implementation of blind sidewalk detection in portable ETA system. In Information Technology and Applications (IFITA) proceedings, 2010 (Vol. 2, pp. 431-434).

Kärcher, S.M., Fenzlaff, S., Hartmann, D., Nagel, S.K., \& Konig, P. (2012). Sensory augmentation for the blind. Front Hum Neurosci 6 .

Kay, L. (1964). An ultrasonic sensing probe as a mobility aid for the blind. Ultrasonics, 2(2), 53-59.

Lenay, C., Gapenne, O., \& Hanneton, S. (2003). Sensory substitution: Limits and perspectives. Touching for Knowing, 275-292.

Levy-Tzedek, S., Hanassy, S., Abboud, S., Maidenbaum, S., \& Amedi, A. (2012). Fast, accurate reaching movements with a visual-to-auditory sensory substitution device. Restor Neurol Neuros, 30(4), 313-323.

Levy-Tzedek, S., Novick, I., Arbel, R., Abboud, S., Maidenbaum, S., Vaadia, E., \& Amedi, A. (2012). Cross-sensory transfer of sensory-motor information: Visuomotor learning affects performance on an audiomotor task, using sensory-substitution. Sci Rep 2 .

Liu, J., Liu, J., Xu, L., \& Jin, W. (2010). Electronic travel aids for the blind based on sensory substitution. In Computer Science and Education (ICCSE) proceedings 2010 (pp. 1328-1331). IEEE.

Loomis, J.M., \& Klatzky, R.L. (2001). Navigating without vision: Basic and applied research. Optometry \& Vision Sci 78(5), 282.

Maidenbaum, S., Abboud, S., \& Amedi, A. (2014). Sensory substitution: Closing the gap between basic research and widespread practical visual rehabilitation. Neurosci Biobehav Rev, 41, 3-15.

Maidenbaum, S., \& Amedi, A. (2012). Applying plasticity to visual rehabilitation in adulthood. Plasticity in sensory systems. Cambridge University Press.

Maidenbaum, S., Arbel, R., Abboud, S., Chebat, D.R., Levy-Tzedek, S., \& Amedi, A. (2012). Virtual 3D shape and orientation discrimination using point distance information. Proc ICDVRAT

Maidenbaum, S., Levy-Tzedek, S., Chebat, D.R., \& Amedi, A. (2013). Increasing accessibility to the blind of virtual environments, using a virtual mobility aid based on the "EyeCane": Feasibility study. PLoS1 8(8), e72555.

Manduchi, R., \& Kurniawan, S. (2010). Watch Your Head, Mind Your Step: Mobility-Related Accidents Experienced by People with Visual Impairment, Tech. Rep. UCSC-SOE-10-24, University of California, Santa Cruz.

Manduchi, R., \& Kurniawan, S. (2011). Mobility-related accidents experienced by people with visual impairment. Research and Practice in Visual Impairment and Blindness, 4(2), 44-54.

Marson, L. (2003). A Study of the Impact of the Rehabilitation Programme Offered by Agencies of the Caribbean Council For the Blind on the Status of Blind and Visually Impaired Women in the, University of the West Indies.
McVea, D.A., \& Pearson, K.G. (2009). Object avoidance during locomotion, Progress in Motor Control, Springer, pp. 293-315.

Meijer, P.B.L. (1992). An experimental system for auditory image representations. IEEE Biomed eng, 39(2), 112-121.

Nau, A.C., Pintar, C., Fisher, C., Jeong, J.H., \& Jeong, K. (2014), A standardized obstacle course for assessment of visual function in ultra low vision and artificial vision. $J$ Vis Exp, 11(84). doi: 10.3791-51205

Pavey, S., Dodgson, A., Douglas, G., \& Clements, B. (2009). Travel, Transport, and Mobility of people who are blind and partially sighted in the UK. Visual Impairment Centre for Teaching and Research, University of Birmingham, RNIB report.

Quinones, P.A., Greene, T., Yang, R., \& Newman, M.W. (2011). Supporting visually impaired navigation: A needs-finding study, ACM Proc CHI, pp. 1645-1650.

Raz, N., Striem, E., Pundak, G., Orlov, T., \& Zohary, U. (2007). Superior serial memory in the blind: A case of cognitive compensatory adjustment. Curr Biol, 17(13), 1129-1133.

Reich, L., Maidenbaum, S., \& Amedi, A. (2012). The brain as a flexible task machine: Implications for visual rehabilitation using noninvasive vs. invasive approaches. Curr Opin Neurol, 25(1), 86-95.

Renier, L., \& De Volder, A.G. (2010). Vision substitution and depth perception: Early blind subjects experience visual perspective through their ears. Disabli Rehabil Assist Technol, 5(3), 175183.

Roentgen, U.R., Gelderblom, G.J., Soede, M., \& Witte, L.D. (2008). Inventory of electronic mobility aids for persons with visual impairments: A literature review. JVIB, 102(11), 702724.

Russell, J.N., Hendershot, G.E., Leclere, F., Howie, L.J., \& Adler, M. (1997). Trends and differential use of assistive technology devices: United States, 1994. US Department of Health and Human Services, Centers for Disease Control and Prevention, National Center for Health Statistics.

Russell, L. (1966). Travel path sounder-further results. In Proc. of the Internat. Conf. on Sensory Dev. for the Blind, R. Dufton (Ed.), London: St. Dunstan's (pp. 293-297).

Ward, J., \& Meijer, P. (2010). Visual experiences in the blind induced by an auditory sensory substitution device. Conscious cog, 19(1), 492-500.

White, G.R., Fitzpatrick, G., \& McAlister, G. (2008). Toward accessible $3 \mathrm{D}$ virtual environments for the blind and visually impaired. ACM Proc DIMEA, 134-141.

Williams, M.D., Ray, C.T., Griffith, J., De L'Aune, W., et al. (2011). The Use of a Tactile-Vision Sensory Substitution System as an Augmentative Tool for Individuals with Visual Impairments. JVIB, 105(1), 45-50.

\section{Supplementary data}

Appendix 1: For a video of how to use the shortsensor of the device see: http://www.youtube.com/ watch? $\mathrm{v}=\mathrm{rpbGaPxUKb} 4$ 
Appendix 2: Some links to publicly available online videos demonstrating the potential of sensory substitution devices:

From the sensory substitution and augmentation conference in the British Academy 2013: http://www.youtube.com/watch?v=6_EJTWeCOuU
The BrainPort: http://www.youtube.com/watch?v= xNkw28fz9u0

The vOICe: $h t t p: / / w w w . y o u t u b e . c o m / w a t c h ? v=S U q$ -vdjRaik 